\title{
Modelling of Oblique Wire Cutting and Experimental Application on Soft Solid Foods for the Investigation of Friction Behaviour
}

\author{
Tilman Witt (D), Yannic Holländer, Sven Tietze, and Jens-Peter Majschak \\ Chair of Processing Machines and Processing Technology, Technische Universität Dresden, 01062 Dresden, Germany \\ Correspondence should be addressed to Tilman Witt; tilman.witt@tu-dresden.de
}

Received 20 February 2019; Accepted 24 April 2019; Published 16 June 2019

Academic Editor: Susana Fiszman

Copyright (c) 2019 Tilman Witt et al. This is an open access article distributed under the Creative Commons Attribution License, which permits unrestricted use, distribution, and reproduction in any medium, provided the original work is properly cited.

\begin{abstract}
The sales value of ready-made foods is determined to a large extent by the appearance of the individual pieces or slices. Breakouts or deformations are perceived negatively and should be avoided. The quality of the cut surface is derived as an evaluation criterion from the evaluation of the cutting force curve. This study examines the influence of friction during cutting. The method developed for this purpose deals with the problem of separating friction from breaking and deforming when cutting with wire. To test the theoretical approach, cutting experiments with thin wires are carried out on various foodstuffs. When cutting with obliquely oriented wire, force value curves are recorded in the feed direction and perpendicular to the feed direction. The cutting force curves are reduced to two characteristic values for the active unit depending on the cross-section geometry. In connection with the result values, an explanatory model is proposed with which a quantified statement on the proportion of friction during the cut is possible. The enhanced model elucidation by oblique cutting will be helpful for the comparison of cutting tools and for the verification of computer simulations.
\end{abstract}

\section{Introduction}

The cutting process of food is influenced by product properties, the geometry of the cutting tool, and the technical parameters of the tool movement. The texture of food is characterised by a low Young's Modulus and often distinctive adhesive behaviour $[1,2]$. Foodstuffs therefore differ significantly from metallic and polymeric materials. The knives for cutting food are of small thickness and a small wedge angle. The oblique cutting movement with a high push-slice ratio prevents crushing of the food [3]. The appearance and roughness of the cut surface is an important quality criterion for foods [4], which is associated with cutting force and sharpness $[5,6]$. The Blade Sharpness Index is determined by the tip radius, the wedge angle, and the blade profile $[7,8]$. In the case of wire cutting, the tip Radii is equivalent to half the wire diameter. After penetration into the material, additional friction occurs between the tool and the sample [1]. Cutting with wire is often used for products where the friction increases rapidly as the contact surface increases. Despite the small surface area of the wire, a residual friction remains [9]. The present study deals with the experimental investigation of wire cutting with oblique wire on foodstuffs. The aim of the present study is the quantitative description of the frictional force that occurs during a cut.

Atkins [10] showed in his investigations on cheese cutting that the interacting surface has a decisive influence on the resulting friction force. The friction coefficient must therefore be set in relation to the interacting surface and the acting normal force. The investigations of Kamyab [9] and Goh [6] deal with the wire cutting of cheese under consideration of friction. Kamyab determines the coefficient of friction by a classical friction test. Goh uses a substitute test from the investigation of Charalambides [11]. In this study, it is assumed that friction can only occur against the direction of motion. This assumption shall be used as an alternative possibility to determine friction during cutting. In the model of Atkins [10, 12], friction is considered in a general equation (1) for work during cutting. 


$$
W=W_{\text {fracture }}+W_{\text {plasticity }}+W_{\text {friction }} .
$$

For food cutting, Dowgiallo [13] replaced the term for plastic deformation by elastic and plastic deformation during cutting. By van Vliet [14], further components for viscous cutting have been introduced. The simplified equation according to Atkins et al. [10] for the pressing cut includes the separation and displacement of the material in one parameter $R$ and the friction in another term. The depth of the material is considered in equation (2) in parameter $w$ and the travelled cutting path $s$. The wire is considered as a line in the model:

$$
\mathrm{d} W=R \cdot w \mathrm{~d} s+\mathrm{d}(\text { friction }) .
$$

There are several studies that have investigated the friction that occurs during the cutting of food $[1,10,15]$. Atkins found that by increasing the angle $\lambda$, the cutting force decreases to a local minimum and then increases

$$
w\left(s_{\text {feed }}\right)=\left\{\begin{array}{l}
w_{\mathrm{S}} \cdot \frac{s_{\text {feed }}-s_{\mathrm{I}}}{\tan \lambda \cdot w_{\mathrm{S}}}, \\
w_{\mathrm{S}} \\
w_{\mathrm{S}} \cdot\left(1-\frac{s_{\mathrm{feed}}-s_{\mathrm{I}}-h_{\mathrm{S}}}{\tan \lambda \cdot w_{\mathrm{S}}}\right),
\end{array}\right.
$$

With $w$, equation (5) for $F_{\mathrm{MD}}$ can be formulated:

$$
F_{\mathrm{MD}}\left(s_{\text {feed }}\right)=R_{\mathrm{MD}} \cdot w\left(s_{\text {feed }}\right) .
$$

Chaiaia [16] postulates in his investigations a hydrostatic core along the cutting edge. The proportion of the hydrostatic pressure on the edge in perpendicular force-accepting direction $F_{\mathrm{CD}}$ requires the reference to projected cutting edge length. The projected cutting edge length is determined in equation (6) by the tangent of the angle $\lambda$ :

$$
F_{\mathrm{CD}}\left(s_{\text {feed }}\right)=R_{\mathrm{CD}} \cdot \tan \lambda \cdot w\left(s_{\text {feed }}\right) .
$$

The angle $\lambda=0^{\circ}$ does not lead to a defined solution for the calculation of $w$ because of the division by zero. The solution of the problem by a case distinction is avoided by the insignificant change from $\lambda=0^{\circ}$ to $\lambda=0.1^{\circ}$. With the equation approach, force curves for $F_{\mathrm{MD}}$ and $F_{\mathrm{CD}}$ are fitted to the measured curves. Figure 2 qualitatively illustrates the force curves for a pressing and an oblique cut.

This model of cutting contains numerous simplifications. The considerations do not take into account the special features of the initial section and the compression present. The material is regarded as rigid in which the cutting tool immediately creates a crack on impact. For the departing of the cutting tool from the material, an occurring brittle fracture or a drag of the material into the countersupport is not mapped by the model. again. This property is attributed to the occurrence of friction. The forces in feed direction $F_{\mathrm{MD}}$ and perpendicular direction $F_{\mathrm{CD}}$ are considered for further investigation of friction. The directions of the forces are shown in Figure 1.

There is no relative movement in the perpendicular direction, which is why instead of mechanical work, the force curves are compared on the basis of the fracture toughness $R$. Equation (2) is therefore reduced to

$$
\mathrm{d} F=R \mathrm{~d} w .
$$

The width to be cut depends on the feed distance $s_{\text {feed }}$ of the wire. By specifying the sample geometry in length $w_{\mathrm{S}}$ and height $h_{\mathrm{S}}$, the calculation of the cut sample width $w$ can be calculated as a function of the feed rate and the point of first contact $s_{\text {I }}$ of the cutting tool with the sample. A division into three sections is necessary for the cutting process. The determination of the section boundaries is demonstrated in

$$
\begin{aligned}
& s_{\mathrm{I}} \leq s_{\text {feed }} \leq s_{\mathrm{I}}+\tan \lambda \cdot w_{\mathrm{S}}, \\
& s_{\mathrm{I}}+\tan \lambda \cdot w_{\mathrm{S}} \leq s_{\text {feed }} \leq s_{\mathrm{I}}+h_{\mathrm{S}}, \\
& s_{\mathrm{I}}+h_{\mathrm{S}} \leq s_{\text {feed }} \leq s_{\mathrm{I}}+h_{\mathrm{S}}+\tan \lambda \cdot w_{\mathrm{S}} .
\end{aligned}
$$

\section{Materials and Methods}

2.1. Test Station for Wire Cutting. The test setup is based on a universal testing machine type Zwick Z020 with a uniaxial feed and is equipped with a force sensor type HP 011562 . The sensor has a measuring range of $\pm 500 \mathrm{~N}$ and detects the force in machine feed direction $F_{\mathrm{MD}}$. Two YCZ-133 bending beams with a measuring range of $\pm 50 \mathrm{~N}$ are used to measure the force orthogonally crossing the feed direction. The measured force in the cross direction $F_{\mathrm{CD}}$ is synchronized with the force $F_{\mathrm{MD}}$ via a light barrier. The arrangement of the sensors within the module installed in the universal testing machine is shown in Figure 3.

The wire can be tensioned into the frame at three different angles $\lambda$. For an angle $\lambda=0^{\circ}$, the wire is orthogonal to the feed direction. Figure 1 shows in position (d) an angle $\lambda=45^{\circ}$ with the solid line and in positions (e) and (f) the angles of $30^{\circ}$ and $0^{\circ}$ as an interrupted line. The specimen lies on the countersupport, which has a gap width of $5 \mathrm{~mm}$. During the test, the countersupport stands still, and the wire with the sensors is moved at 1 or $2 \mathrm{~mm} / \mathrm{s}$ feed rate. All tests are performed in an air-conditioned environment at an ambient temperature of $296 \mathrm{~K}$ and $50 \%$ humidity. The videos V1-V6 in the supplementary material are attached to visualize the experiment. 


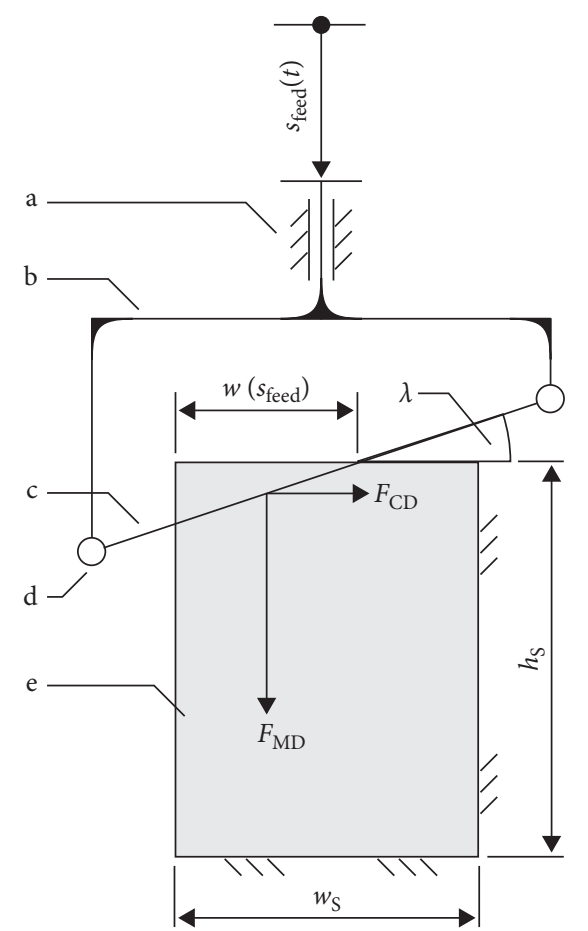

FIGURE 1: Schematic sketch of the cutting test. (a) Uniaxial guidance. (b) Framework for tensioning the wire. (c) Wire. (d) Wire clamping. (e) Specimen. Feed rate as a function of time $s_{\text {feed }}(t)$. Height $h_{\mathrm{S}}$ and width $w_{\mathrm{S}}$ of the sample. Cutting width $w$ as a function of the feed $s_{\text {feed }}$. Oblique angle $\lambda$. The force in feed direction $F_{\mathrm{MD}}$ and the force in orthogonal cross direction $F_{\mathrm{CD}}$.

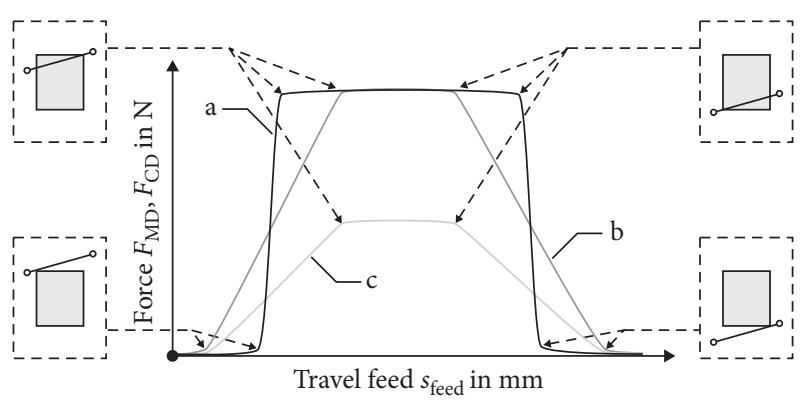

Figure 2: Sketched plot of the calculated force as a function of the feed distance. (a) Describes a typical curve for $F_{\mathrm{MD}}$ with $\lambda=0^{\circ}$. (b) Describes a typical curve for $F_{\mathrm{MD}}$ with $\lambda=45^{\circ}$. (c) Describes a typical curve for $F_{\mathrm{CD}}$ with $\lambda=45^{\circ}$.

\section{Materials}

The experiments are carried out with sausage, Leberkäse, Edam cheese, and bubble gum. The selection of materials is characterised by similar Young's modulus $[2,17]$ and individually distinctive properties. Sausage has a high fat content and fibres that are longer than the diameter of the cutting tool. Leberkäse is finer chopped than sausage and has only occasionally long fibres. Both are characterised by a low Young's modulus compared to metal and have been used in similar investigations [18]. Edam cheese is tested because of its relatively small holes compared to other cheeses and its

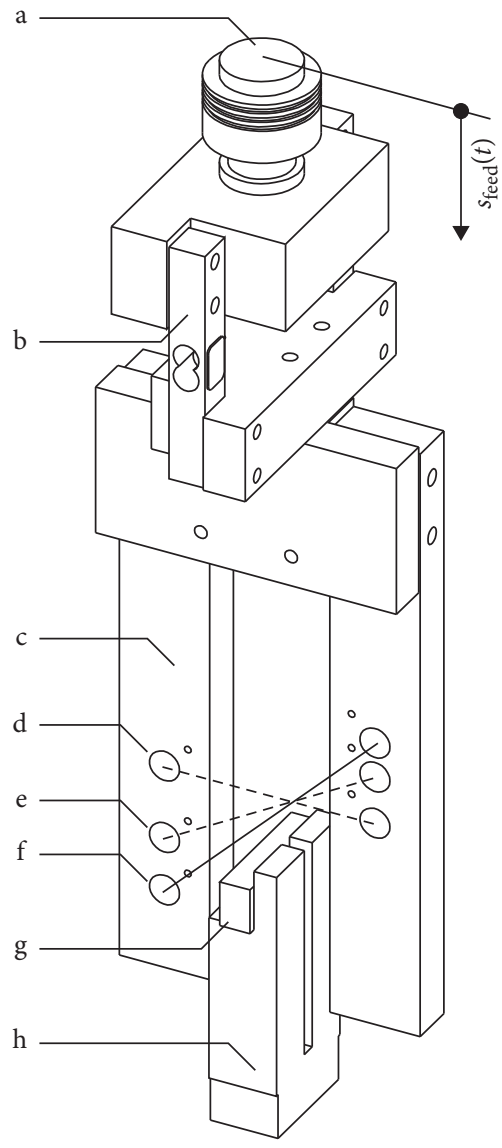

FIGURE 3: Design of the experimental unit. (a) Load cell HP 011562. (b) Bending beams YCZ-133. (c) Frame. (d) Wire fastening position $\lambda=45^{\circ}$. (e) Wire fastening position $\lambda=30^{\circ}$. (f) Wire fastening position $\lambda=0^{\circ}$. (g) Specimen. (h) Countersupport of the sample.

strong tendency to stick to blades [19]. Bubble gum also has a strong tendency to adhere [20], is ductile, and shows a more pronounced viscosity than the other test materials [21]. As the mechanical properties of bubble gum are very temperature-dependent [21], two temperatures will be investigated. Sausage, Leberkäse, and cheese are preconditioned to $281.2 \mathrm{~K}$, while bubble gum is heated to $308.2 \mathrm{~K}$ and $313.2 \mathrm{~K}$, respectively. Due to the two temperatures for bubble gum, there are a total of five different specimens, with bubble gum at $308.2 \mathrm{~K}$ hereinafter referred to as "bubble gum 1 " and bubble gum at $313.2 \mathrm{~K}$ hereinafter referred to as "bubble gum 2". All samples are wrapped and stored for at least four hours at the specified temperature in a cooled incubator. The individual samples shall have a length of at least $30 \mathrm{~mm}$. The cut surface has a width $w_{\mathrm{S}}$ of $10 \mathrm{~mm}$ and different heights depending on the material. For Leberkäse, the samples are $13 \mathrm{~mm}$ high, whereas all other materials have a height $h_{\mathrm{S}}$ of $16 \mathrm{~mm}$.

3.1. Wiresfor Cutting. Two different materials, each with two different diameters, are used for the wires in the experiments. For the diameters, $0.06 \mathrm{~mm}$ and $0.10 \mathrm{~mm}$ molybdenum and dimensions, $0.20 \mathrm{~mm}$ and $0.40 \mathrm{~mm}$ Kanthal are used. Molybdenum has a Young's modulus of elasticity of 
$310 \mathrm{GPa}$ [22] while Kanthal has a Young's modulus of $220 \mathrm{GPa}$ [23]. Kanthal consists of $>69 \% \mathrm{Fe}, 20.5-23.5 \% \mathrm{Cr}$, $\mathrm{Al} 5.8 \%$, and $<0.08 \% \mathrm{C}$. Due to the lower Young's modulus of Kanthal, a comparable elongation is achieved for the thinner wires as for the thicker wires. The wire tension is calculated and adjusted as a function of the first harmonic eigenfrequency. The adjustment and gradual checking of the eigenfrequency is indirectly ensured by striking the string and evaluating the acoustics emitted. In screening tests with a wire diameter of $0.80 \mathrm{~mm}$, strong sample deformations and breakouts from the samples occurred. The experiments are therefore limited to wires with a maximum diameter of $0.4 \mathrm{~mm}$. The choice of wires ensures comparability with the work of Goh [6] and Kamyab [9].

\subsection{Checking and Calibrating the Force Sensors. A significant} measurement uncertainty can be caused by the additional load of a sensor due to lateral force, bending, or torsion. In the test setup described, transverse forces and bending moments occur due to a lever and force components that act perpendicular to the direction of measurement. The accuracy of the sensors is therefore checked by a reference measurement with defined forces and defined force direction. A wire is attached to the middle cutting point of the cutting wire, which leads over a deflection pulley and has a weight of known mass attached to the other end. The cutting wire is moved with the sensors, where the direction of pull of the attached mass changes. The pulley is considered frictionless and the wire massless. The test is carried out at low speed to avoid inertial effects. Figure 4 shows a sketch of the test setup.

The parameter $l_{\text {offset }}$ is set to $200 \mathrm{~mm}$. The stroke $s_{\text {feed }}$ is limited to $600 \mathrm{~mm}$. For the different weights of $20 \mathrm{~g}, 50 \mathrm{~g}$, $100 \mathrm{~g}, 200 \mathrm{~g}$, and $500 \mathrm{~g}$, the test is performed five times each, and the percentage error between expected and measured value is calculated. Figure 5 shows an example of a comparison between the calculated and measured curves of force $F_{\mathrm{MD}}$ and force $F_{\mathrm{CD}}$ for a weight of $100 \mathrm{~g}$. The result of the test is plotted on the basis of a weight of $100 \mathrm{~g}$. The error is calculated as a percentage of the expected and measured value.

The measured force in the feed direction shows a significant difference to the calculated curve for small angles. The relative error between measured value and real value is less than $4 \%$ for forces greater than $0.1 \mathrm{~N}$.

3.3. Consideration of Wire Deflection. The deflection of the wire by the effective force during cutting cannot be avoided in the test setup described. A reduction of the deflection is achieved by increasing the preload of the wire, although the permissible force during cutting is reduced by the faster occurrence of plastic deformation of the wire. To determine the deflection of the wire as a function of the measured force perpendicular to the wire axis, the specimen is replaced by a needle bearing. The wire will deflect on contact with the bearing ring and generate a force on the sensors, as shown in Figure 6.

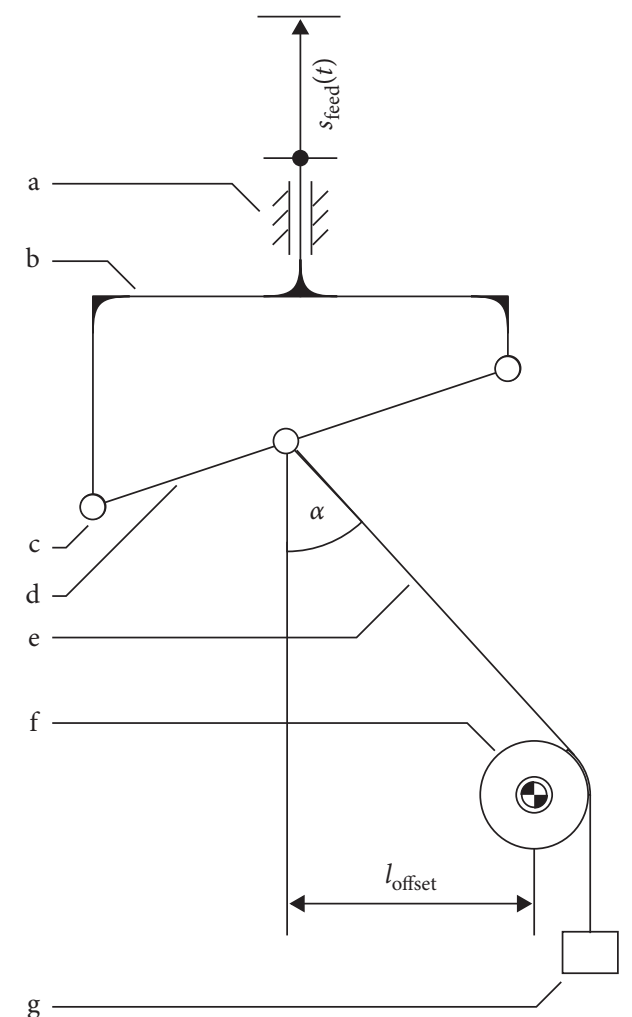

Figure 4: Schematic sketch of the test of the force sensors. (a) Uniaxial guidance. (b) Framework for tensioning the wire. (c) Thick wire. (d) Clamping wire. (e) Thinner wire. (f) Pulley. (g) Test mass. Angle of the acting force in relation to the feed direction $\alpha$. Feed as a function of time $s_{\text {feed }}(t)$. Distance between pulley pivot point and feed axis $l_{\text {offset }}$. Test mass $m$ attached to a wire.

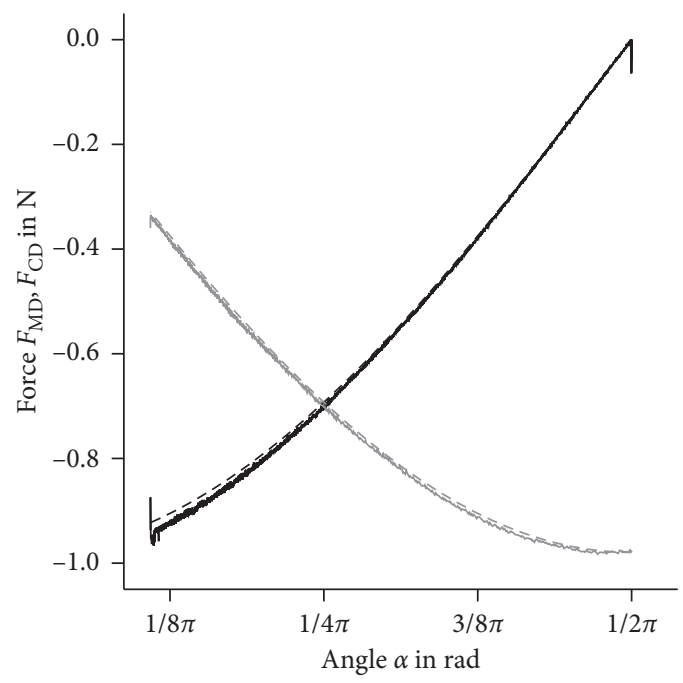

FIgURE 5: Plot of the measured forces with respect to the feed distance over $600 \mathrm{~mm}$ path at $100 \mathrm{~g}$ weight. In black is $F_{\mathrm{MD}}$ and in grey $F_{\mathrm{CD}}$. The solid lines represent the measured forces and the dotted lines represent the calculated forces.

For each combination of wire diameter and oblique angle, five repetitions of the test are performed and a correction curve is derived. Using the curve, the true position of 


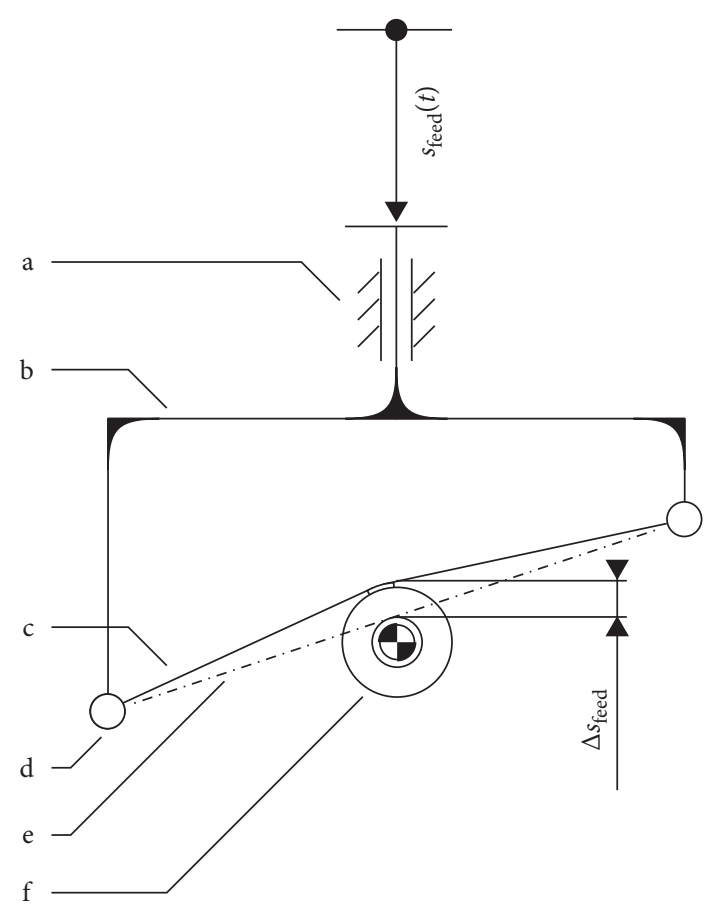

FIGURE 6: Schematic sketch of the wire deflection test. (a) Uniaxial guidance. (b) Framework for tensioning the wire. (c) Wire deflected. (d) Wire clamping. (e) Wire not deflected. (f) Pulley. Feed travel $s_{\text {feed }}(t)$. Wire deflection $\Delta s_{\text {feed }}$.

the wire in the product is calculated back to the path identification by the feed axis. Figure 7 shows the correction for a selected example.

The corrected force curves occur at a wire deflection of $0 \mathrm{~mm}$. A residual error in the correction remains due to the averaging of five repetitions and leads to minor deviations. The maximum deflection is determined at a wire diameter of $0.06 \mathrm{~mm}$ and an angle $\lambda=45^{\circ}$. The deflection of the wire is determined by the force vector in perpendicular direction to the wire axis. A map for the compensation of the deflection depending on the perpendicular force vector to the wire is established after this experiment. The data are used to correct the measured cutting force curves in order to obtain a force curve that is as representative as possible.

\section{Results}

4.1. Comparison of Model and Cutting Force Curve. The performance of the tests with sausage shows very large differences in the cutting forces between the individual samples. Sausage is therefore not considered in the further evaluation. The remaining test specimens show comparable behaviour at similar cutting forces. The comparison of the measured force curve with the fitted force curve is shown below. The point of initial contact between wire and specimen is determined according to the criterion of a force threshold. By the known height and width of the specimen and the angle $\lambda$, the interacting length $w$ is determined for each waypoint according to equation (4). The force curves should be approximated by equations (5) and (6). A fit according to the criterion of the smallest error squares is

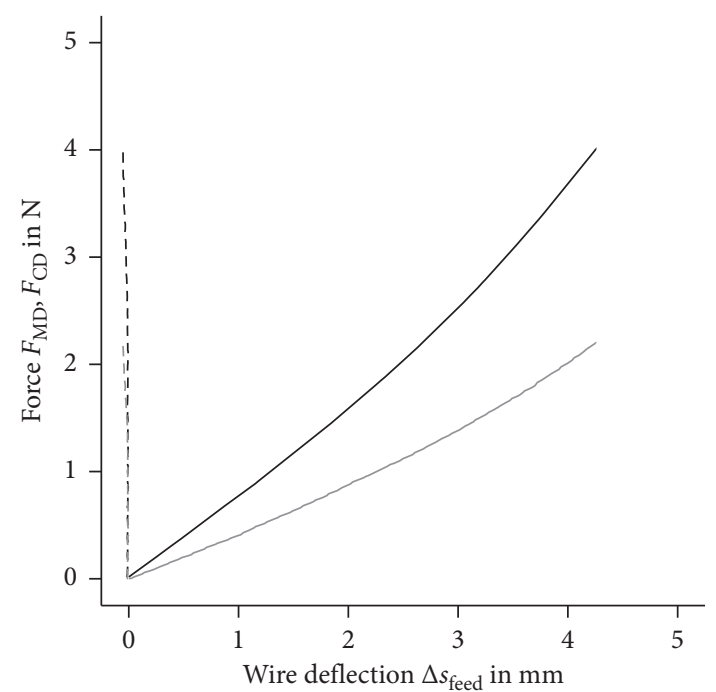

Figure 7: Plot of forces with respect to the feed difference with $\lambda=30^{\circ}$. The black continuous and dotted lines represent $F_{\mathrm{MD}}$ for measured and corrected trace. The grey solid and dotted lines represent $F_{\mathrm{CD}}$ for the measured and corrected trace.

used to obtain the characteristic parameters $R_{\mathrm{MD}}$ and $R_{\mathrm{CD}}$. Figure 8 shows the force curves of the four remaining materials next to each other with the same settings.

The results of the fitting according to Figure 8 are for $R_{\mathrm{MD}}$ and $R_{\mathrm{CD}}$ values of $77 \mathrm{~J} / \mathrm{m}^{2}$ and $56 \mathrm{~J} / \mathrm{m}^{2}$ for cheese, $114 \mathrm{~J} / \mathrm{m}^{2}$ and $79 \mathrm{~J} / \mathrm{m}^{2}$ for Leberkäse, $133 \mathrm{~J} / \mathrm{m}^{2}$ and $55 \mathrm{~J} / \mathrm{m}^{2}$ for bubble gum 1 , and $84 \mathrm{~J} / \mathrm{m}^{2}$ and $39 \mathrm{~J} / \mathrm{m}^{2}$ for bubble gum 2 . The values for all tests are listed in the supplementary file. The differences between the measured and calculated curve are smaller for cheese in Figure 8 than for the other materials. Leberkäse follows the principle course of the curve with force jumps being visible. In the observation of the experiments, an irregular speed of the wire can be seen while passing through, which can also be seen in the video. Shortly before the end of the cut, the remaining material ruptures, which can be seen in the force curve due to an abrupt drop in force. The bubble gums show larger deviations from the calculated curve when the wire exits. From the observations of the slip a stronger pulling of the material into the countersupport is recognizable. The statistical comparison of the model and the measurement is illustrated by the standard error of the estimation $S_{\text {Est }}$. Figure 9 shows the error values for the feed direction in black and the error values for the perpendicular cross direction in grey. The different angles are distinguished by different markers.

The standard error of estimate for Edam cheese, Leberkäse and bubble gum 1 is significantly greater for $\lambda=0^{\circ}$ than for $\lambda=30^{\circ}$ and $45^{\circ}$ with the same wire diameter. For bubble gum 2 this difference cannot be shown. For the respective materials and diameters for $\lambda=30^{\circ}$ or $45^{\circ}$ the errors are on a similar level. Bubble gum 1 has an overall higher average of defects. Edam cheese has increasing errors with increasing wire diameter, while Leberkäse shows this tendency only slightly for the transverse force. As the wire diameter increases, the force error in bubble 


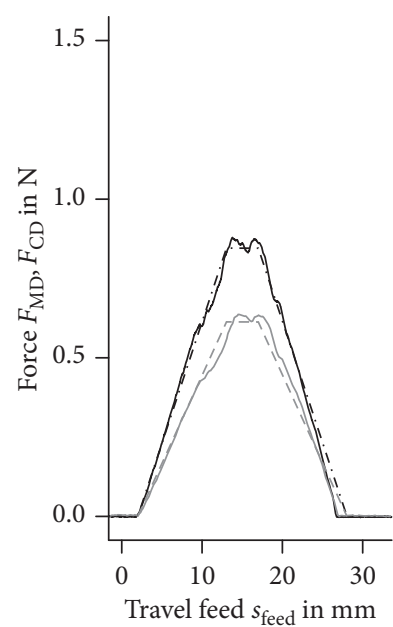

(a)

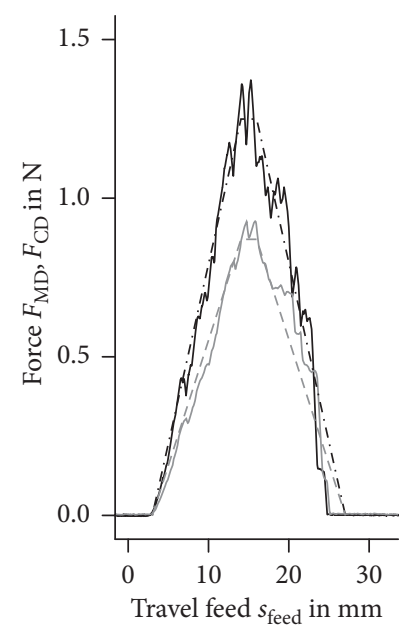

(b)

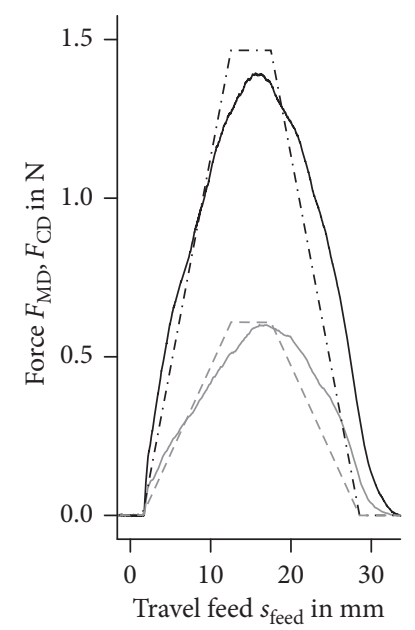

(c)

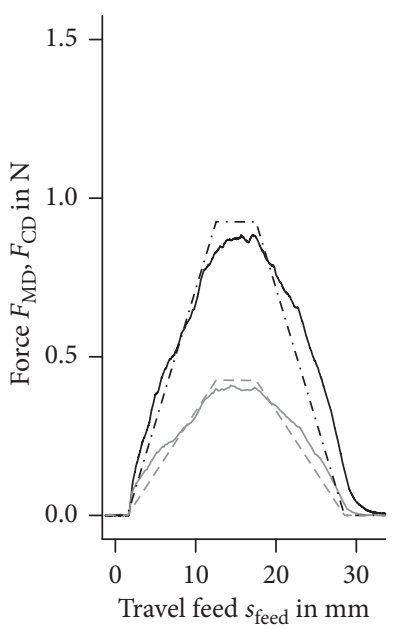

(d)

FIgUre 8: Plot the force in respect to the feed during a cut. The angle $\lambda=45^{\circ}$ and the wire has a diameter of $d_{\text {Wire }}=0.1 \mathrm{~mm}$. The feed rate $v_{\text {speed }}=1 \mathrm{~mm} / \mathrm{s}$. The $F_{\mathrm{MD}}$ is shown in black and the $F_{\mathrm{CD}}$ in grey. The dash-dot lines represent the fitted force curves and the continuous lines result from the corrected measurements. (a) Edam Cheese. (b) Leberkäse. (c) Bubble gum 1. (d) Bubble gum 2.

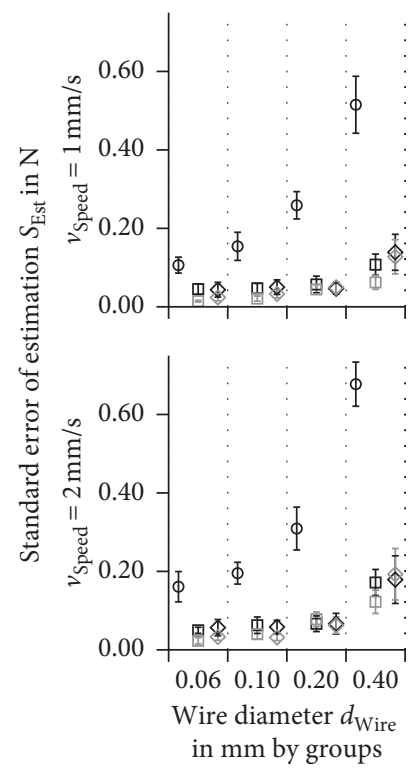

(a)

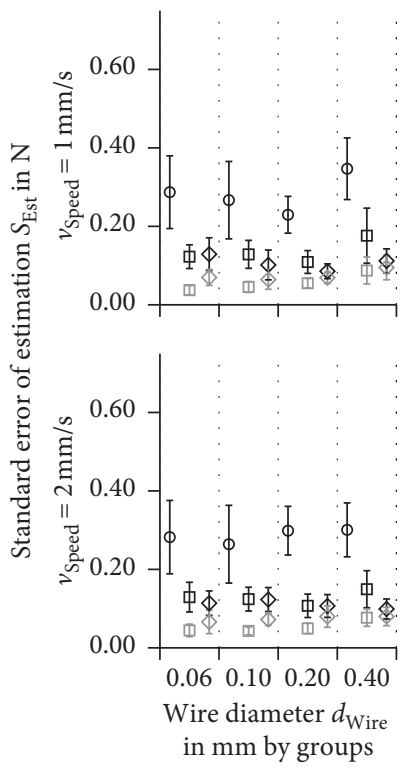

(b)

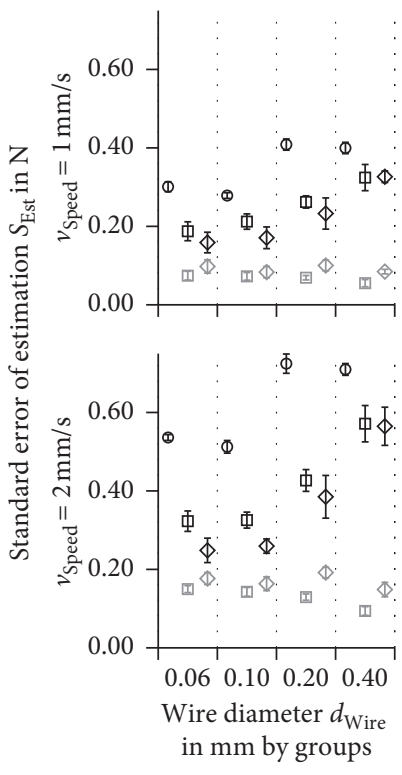

(c)

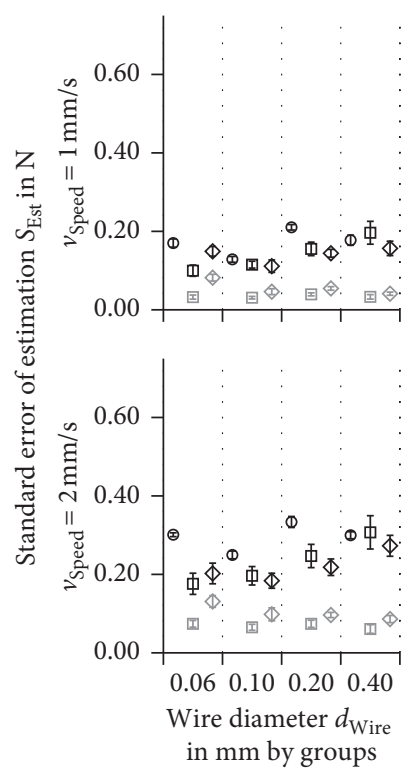

(d)

FigURE 9: Plot of the standard error of estimation $S_{\text {Est }}$ in respect to the wire diameter $d_{\text {Wire. }}$ The error for $F_{\mathrm{MD}}$ is shown in black and for $F_{\mathrm{CD}}$ in grey. The circles represent $\lambda=0^{\circ}$, rectangles for $\lambda=30^{\circ}$, and rhombus for $\lambda=45^{\circ}$. The error bars represent the standard deviation of measurements. (a) Edam Cheese. (b) Leberkäse. (c) Bubble gum 1. (d) Bubble gum 2.

gum increases in the feed direction, while the force error in the transverse direction decreases. An increase of the standard error by increasing the feed rate can be observed especially with bubble gum. Edam cheese and Leberkäse, on the other hand, show only slight increases. The lowtemperature bubble gum 1 has a larger mean standard error than the high-temperature bubble gum 2. The standard deviation of the standard error of estimate increases with increasing standard error. For Leberkäse, the standard deviation relative to the standard error is higher than for the other materials.
4.2. Fracture Toughness in Feed Direction and Perpendicular Direction. The fitted values for the factors $R_{\mathrm{MD}}$ and $R_{\mathrm{CD}}$ are shown in Figure 10 as a function of the feed rate $v_{\text {speed }}$, the oblique angle $\lambda$, the wire diameter $d_{\text {Wire }}$, and the material. A meaningful evaluation of $R_{\mathrm{CD}}$ is not possible for press cuts. Therefore, only $R_{\mathrm{MD}}$ is displayed for $\lambda=0^{\circ}$.

The characteristic factors $R_{\mathrm{MD}}$ and $R_{\mathrm{CD}}$ for the cutting force curves are analysed with a regression for the influencing variables. A mixed model that considers the material as a random intercept is discarded due to poor elucidation. Accordingly, a single model is generated for each material. 


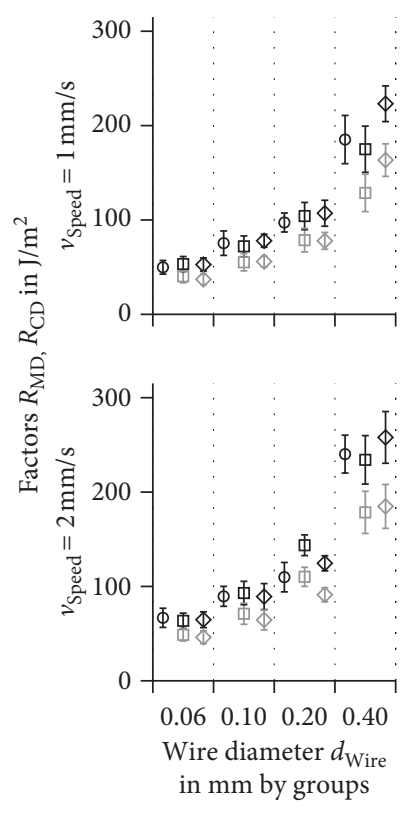

(a)

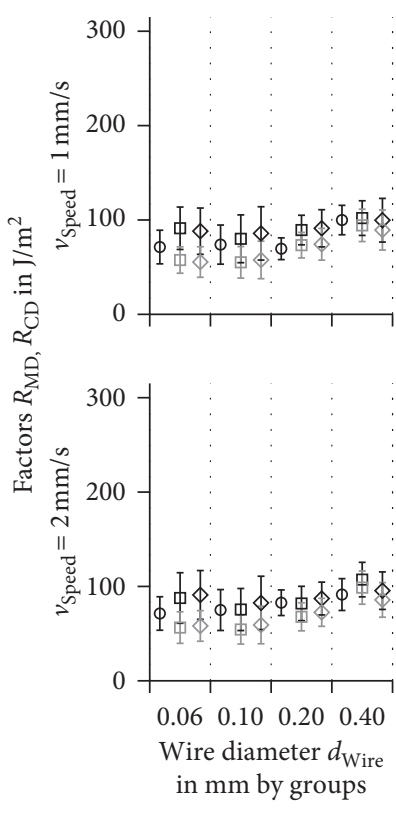

(b)

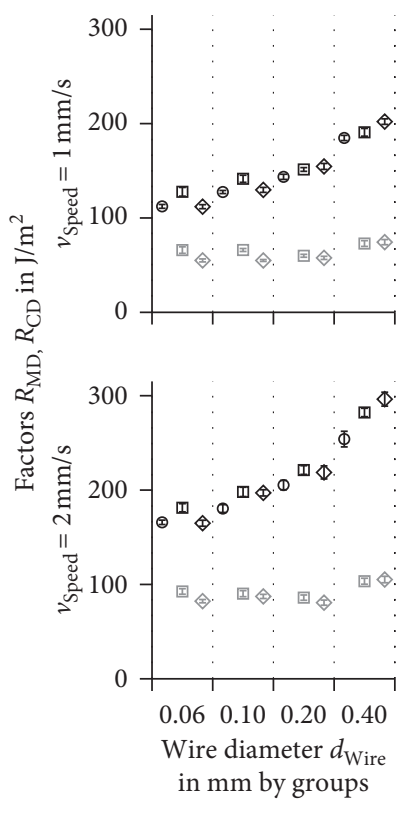

(c)
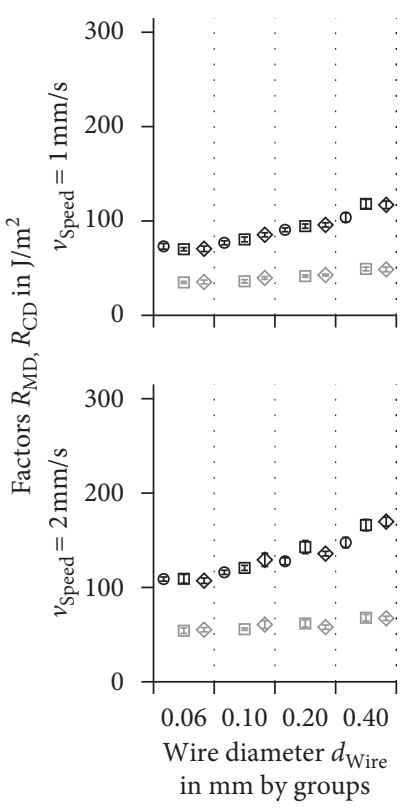

(d)

Figure 10: Plot of the factors of the regression $R_{\mathrm{MD}}, R_{\mathrm{CD}}$ in relation to the wire diameters $d_{\text {Wire. }}$ The values for $R_{\mathrm{MD}}$ are shown in black and for $R_{\mathrm{CD}}$ in grey. The circles represent $\lambda=0^{\circ}$, rectangles for $\lambda=30^{\circ}$, and rhombus for $\lambda=45^{\circ}$. The error bars represent the standard deviation of measurements. (a) Edam Cheese. (b) Leberkäse. (c) Bubble gum 1. (d) Bubble gum 2.

Equation (7) shows the model for the value of $R_{\mathrm{MD}}$ created for each material:

$$
\begin{aligned}
& R_{\text {MD Material }}\left(\lambda, v_{\text {Speed }}, d_{\text {Wire }}\right) \\
& \quad=\left(\text { Intercept }+\frac{\beta_{\lambda}}{\mathrm{rad}} \cdot \lambda+\frac{\beta_{\mathrm{v}} \cdot s}{\mathrm{~mm}} \cdot v_{\text {Speed }}+\frac{\beta_{\mathrm{d}}}{\mathrm{mm}} \cdot d_{\text {Wire }}\right) \mathrm{J} / \mathrm{m}^{2} .
\end{aligned}
$$

The regression coefficients and their significance are listed in Table 1. The significance is given in steps, where the $p$ value must be less than 0.001 for three stars and less than 0.01 for two stars. For the regression, the influences are scaled to the range from 0 to 1 in order to be able to compare the effect strength.

All oblique angles are taken into account when determining $R_{\mathrm{MD}}$, whereas for $R_{\mathrm{CD}}$ the pressing cut is not taken into account. The recorded influencing variables are significant for $R_{\mathrm{MD}}$ in almost all cases. The influence of speed in Leberkäse is not significant for any of the factors. The influence of the oblique angles is not significant for $R_{\mathrm{CD}}$ in several cases. The quantitative values for the coefficients reflect the expected increases according to Figure 10. The scaled coefficients of the oblique angles are significantly lower compared to the other coefficients.

\section{Discussion}

The increasing standard errors of estimate with increasing wire diameter are due to a stronger deformation of the cutting quality at the entry and depart of the wire. This applies in particular to the pressing cut, in which the wire rests on the entire line and the material is strongly deformed before the cut is initiated. Bubble gum shows the largest overall deviations. Experiments have shown that chewing gum exhibits pronounced ductile behaviour compared to other materials. Both the ductile and the elastic behaviour are not represented by the regression and therefore lead to higher standard errors.

Other studies have shown that Edam cheese and bubble gum have high friction, whereas Leberkäse has relatively low friction. The comparison of $R_{\mathrm{MD}}$ with $R_{\mathrm{CD}}$ for Leberkäse shows an approximate equality of both factors. Edam cheese, on the other hand, shows a colinear increase in $R_{\mathrm{CD}}$ as $R_{\mathrm{MD}}$ increases. Colinearity cannot be assumed for bubble gum, which also has high friction. For Edam cheese and bubble gum, there is a clear difference between $R_{\mathrm{MD}}$ and $R_{\mathrm{CD}}$. It is therefore assumed that this difference can be explained by the occurrence of friction. Figure 11 sketches the force ratios for a cut with an oblique wire.

It is assumed that a surface load can be indicated on the surface of the round cutting tool as a function of the angle $q(\varphi)$. Without loss of generality, the representation is selected for a specific $\varphi_{0}$. The value for $q\left(\varphi_{0}\right)$ is expressed as normal force $\mathrm{d} F_{\mathrm{N}}\left(\varphi_{0}\right)$ for illustration purposes. The particles of the test material move in a plane that can be determined by the coordinates of the contact point between the cutting tool and the test material and the tangential plane at the contact point on the surface of the cutting tool. In Figure 11, the plane is given by the vectors $c_{\text {Wire }}$ and $t_{\text {Wire }}$ and the contact point. Perpendicular to the plane in the contact point, the force $\mathrm{d} F_{\mathrm{N}}\left(\varphi_{0}\right)$ is shown. The 
TABle 1: Model influences with significance rating.

\begin{tabular}{|c|c|c|c|c|c|c|c|c|c|}
\hline & \multirow{2}{*}{$\begin{array}{c}\text { Coefficient } \\
\text { Intercept }\end{array}$} & \multicolumn{2}{|c|}{ Edam cheese } & \multicolumn{2}{|c|}{ Leberkäse } & \multicolumn{2}{|c|}{ Bubble gum 1} & \multicolumn{2}{|c|}{ Bubble gum 2} \\
\hline \multirow{4}{*}{$R_{\mathrm{MD}}$ in $\mathrm{J} / \mathrm{m}^{2}$} & & -12.79 & $* *$ & 71.24 & $* * *$ & 25.70 & $* * *$ & 17.80 & $* * *$ \\
\hline & $\beta_{\lambda}$ & 12.21 & $* * *$ & 14.47 & $* * *$ & 17.99 & $* * *$ & 10.97 & $* * *$ \\
\hline & $\beta_{\mathrm{v}}$ & 25.33 & $* * *$ & -1.04 & & 65.60 & $* * *$ & 42.05 & $* * *$ \\
\hline & $\beta_{\mathrm{d}}$ & 464.33 & $* * *$ & 54.22 & $* * *$ & 257.95 & $* * *$ & 131.84 & $* * *$ \\
\hline \multirow{4}{*}{$R_{\mathrm{CD}}$ in $\mathrm{J} / \mathrm{m}^{2}$} & Intercept & -9.47 & & 51.02 & $* * *$ & 39.72 & $* * *$ & 12.96 & $* * *$ \\
\hline & $\beta_{\lambda}$ & 4.58 & & -2.62 & & -18.89 & $* * *$ & 2.83 & \\
\hline & $\beta_{\mathrm{v}}$ & 19.77 & $* * *$ & -0.47 & & 27.60 & $* * *$ & 19.09 & $* * *$ \\
\hline & $\beta_{\mathrm{d}}$ & 349.10 & $* * *$ & 109.29 & $* * *$ & 44.27 & $* * *$ & 37.35 & $* * *$ \\
\hline
\end{tabular}

${ }^{* * *} p<0.001 ;{ }^{* *} p<0.01$

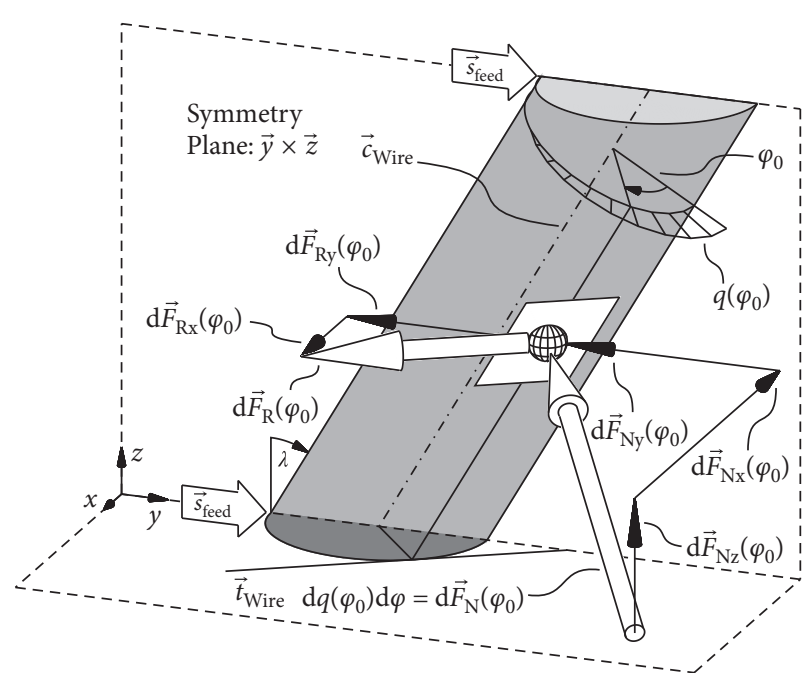

FIgURE 11: Half symmetrical sketch of the acting forces and their Cartesian components on the surface of an oblique wire. The angle of the slope is indicated by $\lambda$. The feed direction is represented by $s_{\text {feed. }}$. The line load $q\left(\varphi_{0}\right)$ describes the load along the wire circumference. For a specific $\varphi_{0}$, the normal force $\mathrm{d} F_{\mathrm{N}}\left(\varphi_{0}\right)$ at the incremental contact point is represented in perpendicular direction to the wire surface. The tangential wire surface is described by the tangent $t_{\text {Wire }}$ and the rotation axis of the wire $c_{\text {Wire }}$. The friction force $\mathrm{d} F_{\mathrm{R}}$ acts in the direction of $t_{\text {Wire }}$. The forces $\mathrm{d} F_{\mathrm{N}}\left(\varphi_{0}\right)$ and $\mathrm{d} F_{\mathrm{R}}\left(\varphi_{0}\right)$ are also given in their Cartesian components.

direction of the feed $s_{\text {feed }}$ forces the particle to move in the tangential plane. Since there are other particles above and below the particle, the movement on the surface will be normal to the cutting surface. The normal vector of the cutting surface $e_{\mathrm{x}}$, the vector in feed direction $s_{\text {feed }}$, and the contact point define the plane of motion. The intersection of the tangential plane and the motion plane of the particle results in the motion line of the particle. The line of motion is parallel to the displayed tangent $t_{\text {Wire }}$. The force vector $\mathrm{d} F_{\mathrm{R}}\left(\varphi_{0}\right)$ is located on this line. The splitting of the force vectors along the Cartesian coordinate system allows the directional summation of the force components. The components in $x$ direction cancel each other out due to symmetry. The part in $z$ direction is generated exclusively by the normal force, whereas the force in $y$ direction is influenced by normal force and friction force. Formulas (8)-(10) summarize the dependencies for the $x, y$, and $z$ directions:

$$
\begin{aligned}
F_{\mathrm{x}}= & F_{\mathrm{Nx}}+F_{\mathrm{Rx}}=\int_{0}^{\pi} q(\varphi) \cdot \sin \varphi \mathrm{d} \varphi \\
& +\int_{0}^{\pi} q(\varphi) \cdot \mu \cdot \sin \left(\arctan \left(\frac{\tan \varphi}{\cos \lambda}\right)\right) \mathrm{d} \varphi, \\
F_{\mathrm{y}}= & F_{\mathrm{Ny}}+F_{\mathrm{Ry}}=\cos \lambda \cdot \int_{0}^{\pi} q(\varphi) \cdot \cos \varphi \mathrm{d} \varphi \\
& +\int_{0}^{\pi} q(\varphi) \cdot \mu \cdot \cos \left(\arctan \left(\frac{\tan \varphi}{\cos \lambda}\right)\right) \mathrm{d} \varphi, \\
F_{\mathrm{z}}= & F_{\mathrm{Nz}}=\sin \lambda \cdot \int_{0}^{\pi} q(\varphi) \cdot \cos \varphi \mathrm{d} \varphi .
\end{aligned}
$$

An evaluation in $x$ direction is not possible due to the symmetry. Since $q(\varphi)$ and friction coefficient $\mu$ are unknown, the integral parts in equations (11) and (12) are substituted by $F_{\text {fric }}$ and $F_{\text {fracdisp. }}$.

$$
\begin{aligned}
& F_{y}=\cos \lambda \cdot F_{\text {fracdisp }}+F_{\text {fric }}, \\
& F_{z}=\sin \lambda \cdot F_{\text {fracdisp }} .
\end{aligned}
$$

The friction force $F_{\text {fric }}$ and the force corresponding to fraction and displacement $F_{\text {fracdisp }}$ can be determined if $F_{\mathrm{y}}$ and $F_{\mathrm{z}}$ are known. The force values were recorded in the experiments. An equation of the force in motion righting by the equation of the experiment 5 and equation of the model approach 12 can be converted to equation (13). The same applies to perpendicular to the direction of motion by equating equations (6) and (12) to equation (14).

$$
\begin{aligned}
F_{\text {fric }} & =R_{\mathrm{MD}} \cdot w\left(s_{\text {feed }}\right)-\cos \lambda \cdot F_{\text {fracdisp }}, \\
F_{\text {fracdisp }} & =R_{\mathrm{CD}} \cdot \frac{1}{\cos \lambda} \cdot w\left(s_{\text {feed }}\right) .
\end{aligned}
$$

The methodical procedure enables the comparison between active units with regard to their friction behaviour. The quantitative evaluation during the cut has the advantage that the friction can also be determined for normal forces at which the material to be cut is destroyed. The method thus represents a possibility for determining the friction. Computer simulations are recommended for further investigation and confirmation of the methodological approach. 


\section{Conclusions}

The additional recording of the force curve in orthogonal direction to the cutting direction enables the evaluation of a second equation and a higher clarification in the model approach for the description of cutting. The implementation and evaluation of a test series provides quantitative values for testing the discussed model approach. The limits of the model are investigated in experiments, whereby especially materials with high tensile strength and low complex modulus are insufficiently represented. The proportion of friction during cutting can be determined by evaluating the force curve in the feed direction and the force curve in the perpendicular direction. The methodical approach makes it possible to identify measures for reducing the cutting forces associated with an increase in cutting quality.

\section{Data Availability}

The recorded characteristic values used to support the findings of this study are included within the supplementary information files.

\section{Conflicts of Interest}

The authors declare that there are no conflicts of interest regarding the publication of this paper.

\section{Acknowledgments}

This work was supported by funds from the European Social Fund and cofinanced by tax revenues on the basis of the budget approved by the members of the Saxon State Parliament and by funds from Theegarten-Pactec $\mathrm{GmbH} \& \mathrm{Co}$. KG. We acknowledge the support by the Open Access Publication Funds of the SLUB/TU Dresden.

\section{Supplementary Materials}

The characteristic test values per section are provided as a list in a CSV file. Six videos of the cuts are included to visualize the experiments. (Supplementary Materials)

\section{References}

[1] Y. Schneider, S. Zahn, C. Schindler, and H. Rohm, "Ultrasonic excitation affects friction interactions between food materials and cutting tools," Ultrasonics, vol. 49, no. 6-7, pp. 588-593, 2009.

[2] C. Schmidt, R. Bornmann, S. Schuldt, Y. Schneider, and H. Rohm, "Thermo-mechanical properties of soft candy: application of time-temperature superposition to mimic response at high deformation rates," Food Biophysics, vol. 13, no. 1, pp. 11-17, 2018.

[3] T. Atkins, "Optimum blade configurations for the cutting of soft solids," Engineering Fracture Mechanics, vol. 73, no. 16, pp. 2523-2531, 2006.

[4] Y. Schneider, S. Zahn, and L. Linke, "Qualitative process evaluation for ultrasonic cutting of food," Engineering in Life Sciences, vol. 2, no. 6, pp. 153-157, 2002.
[5] A. G. Atkins and J. F. V. Vincent, "An instrumented microtome for improved histological sections and the measurement of fracture toughness," Journal of Materials Science Letters, vol. 3, no. 4, pp. 310-312, 1984.

[6] S. M. Goh, M. N. Charalambides, and J. G. Williams, "On the mechanics of wire cutting of cheese," Engineering Fracture Mechanics, vol. 72, no. 6, pp. 931-946, 2005.

[7] G. A. Reilly, B. A. O. McCormack, and D. Taylor, "Cutting sharpness measurement: a critical review," Journal of Materials Processing Technology, vol. 153-154, pp. 261-267, 2004.

[8] C. T. McCarthy, A. N. Annaidh, and M. D. Gilchrist, "On the sharpness of straight edge blades in cutting soft solids: part II-analysis of blade geometry," Engineering Fracture Mechanics, vol. 77, no. 3, pp. 437-451, 2010.

[9] I. Kamyab, S. Chakrabarti, and J. G. Williams, "Cutting cheese with wire," Journal of Materials Science, vol. 33, no. 11, pp. 2763-2770, 1998.

[10] A. G. Atkins, X. Xu, and G. Jeronimidis, "Cutting, by 'pressing and slicing,' of thin floppy slices of materials illustrated by experiments on cheddar cheese and salami," Journal of Materials Science, vol. 39, no. 8, pp. 2761-2766, 2004.

[11] M. N. Charalambides, S. M. Goh, S. L. Lim, and J. G. Williams, "The analysis of the frictional effect on stress-strain data from uniaxial compression of cheese," Journal of Materials Science, vol. 36, no. 9, pp. 2313-2321, 2001.

[12] A. G. Atkins, "Fracture toughness and cutting," International Journal of Production Research, vol. 12, no. 2, pp. 263-274, 1974.

[13] A. Dowgiallo, "Cutting force of fibrous materials," Journal of Food engineering, vol. 66, no. 1, pp. 57-61, 2005.

[14] T. van Vliet, "Large deformation and fracture behaviour of gels," Current Opinion in Colloid \& Interface Science, vol. 1, no. 6, pp. 740-745, 1996.

[15] T. Brown, S. J. James, and G. L. Purnell, "Cutting forces in foods: experimental measurements," Journal of food engineering, vol. 70, no. 2, pp. 165-170, 2005.

[16] B. Chiaia, "Fracture mechanisms induced in a brittle material by a hard cutting indenter," International Journal of Solids and structures, vol. 38, no. 44-45, pp. 7747-7768, 2001.

[17] K. R. Agrawal, P. W. Lucas, J. F. Prinz, and I. C. Bruce, "Mechanical properties of foods responsible for resisting food breakdown in the human mouth," Archives of Oral Biology, vol. 42, no. 1, pp. 1-9, 1997.

[18] S. Schuldt, Y. Schneider, and H. Rohm, "High-speed cutting of foods: cutting behavior and initial cutting forces," Journal of Food Engineering, vol. 230, pp. 55-62, 2018.

[19] G. Arnold, L. Leiteritz, S. Zahn, and H. Rohm, "Ultrasonic cutting of cheese: composition affects cutting work reduction and energy demand," International Dairy Journal, vol. 19, no. 5, pp. 314-320, 2009.

[20] K. Nevzat, I. Palabiyik, S. O. Toker, and O. Sagdic, "Chewing gum: production, quality parameters and opportunities for delivering bioactive compounds," Trends in Food Science \& Technology, vol. 55, pp. 29-38, 2016.

[21] S. Schuldt, T. Witt, C. Schmidt et al., "High-speed cutting of foods: development of a special testing device," Journal of Food Engineering, vol. 216, pp. 36-41, 2018.

[22] R. Farraro and R. B. Mclellan, "Temperature dependence of the Young's modulus and shear modulus of pure nickel, platinum, and molybdenum," Metallurgical Transactions A, vol. 8, no. 10, pp. 1563-1565, 1977.

[23] A. Saigal and G. G. Leisk, "Residual strains and stresses in tungsten/kanthal composites," Materials Science and Engineering: $A$, vol. 237, no. 1, pp. 65-71, 1997. 


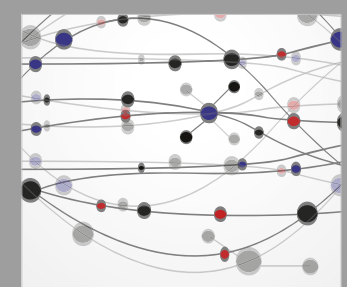

The Scientific World Journal
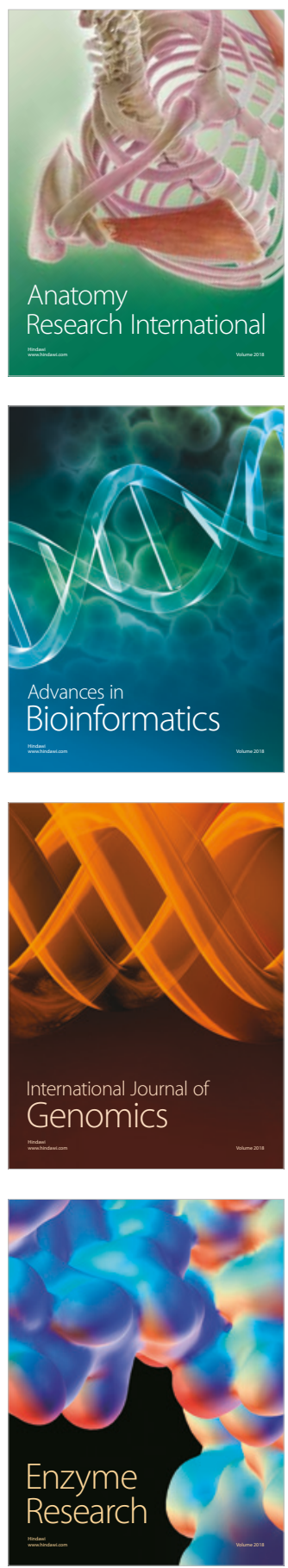
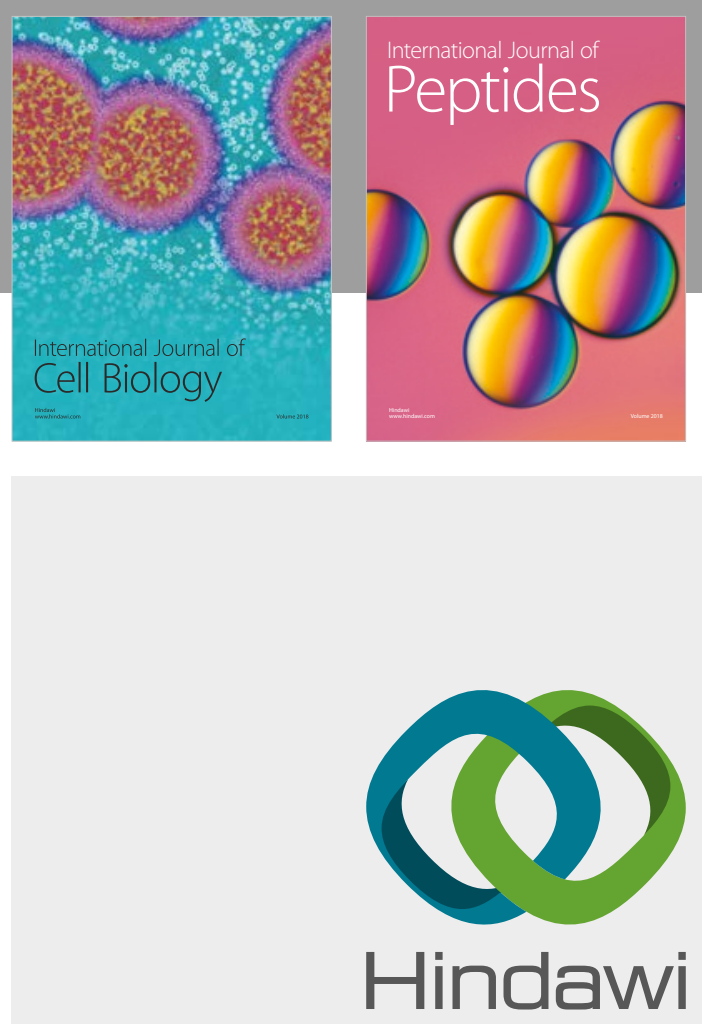

Submit your manuscripts at

www.hindawi.com
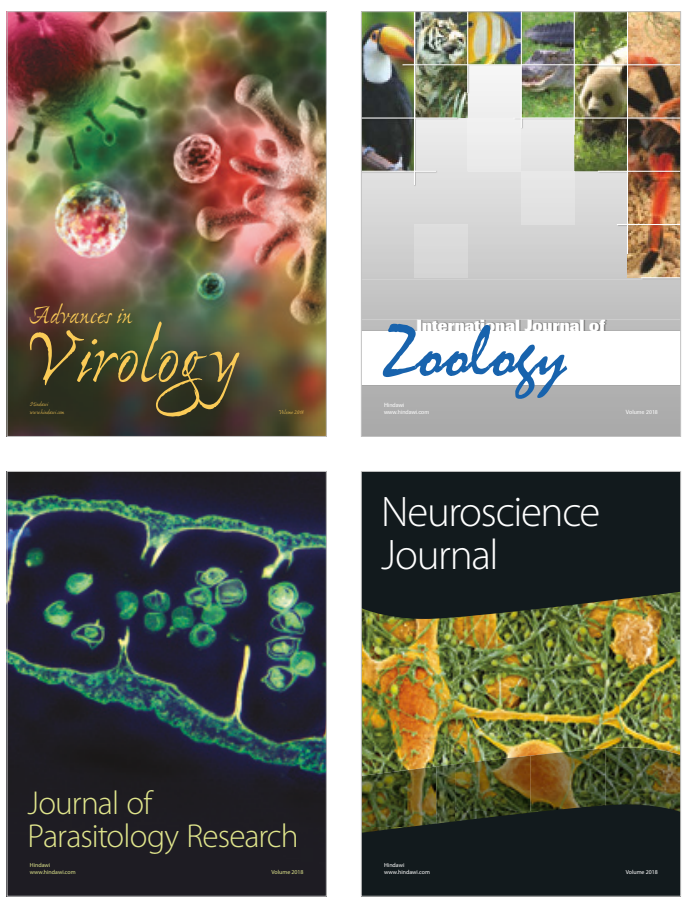
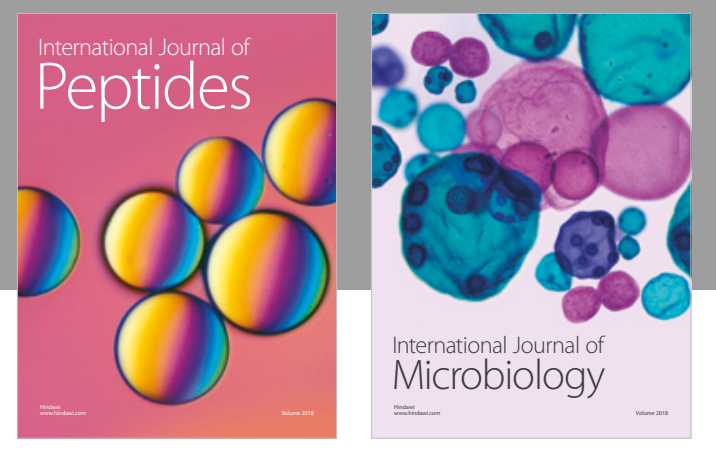

nternational Journal of Microbiology
Journal of
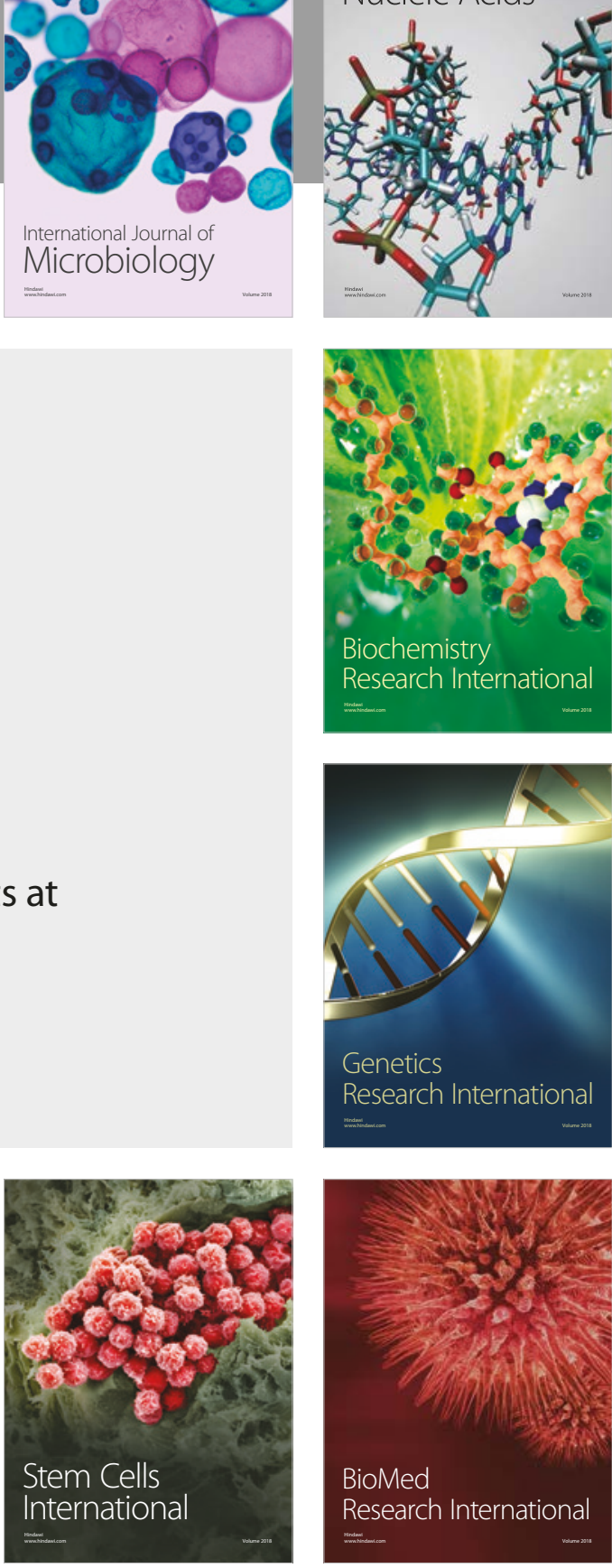
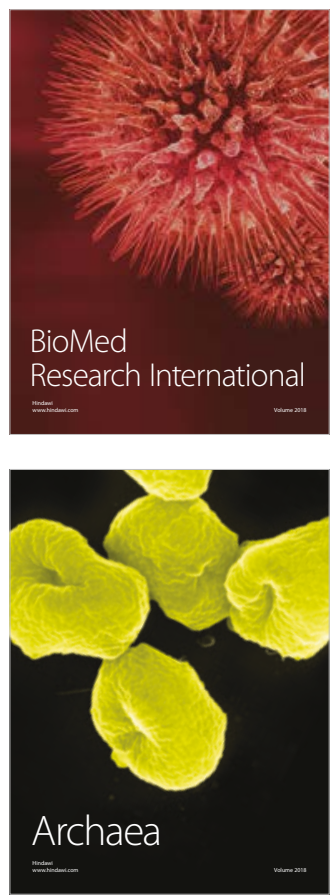